\title{
Replacing sugary drinks with milk is inversely associated with weight gain among young obesity-predisposed children
}

\author{
Miaobing Zheng ${ }^{1 *}$, Anna Rangan ${ }^{1}$, Margaret Allman-Farinelli ${ }^{1}$, Jeanett Friis Rohde ${ }^{2}$, Nanna Julie Olsen ${ }^{2}$ \\ and Berit Lilienthal Heitmann ${ }^{2,3,4}$ \\ ${ }^{1}$ School of Molecular Bioscience, Charles Perkins Centre, University of Sydney, Sydney, NSW 2006, Australia \\ ${ }^{2}$ Research Unit for Dietary Studies, Institute of Preventive Medicine, Bispebjerg and Frederiksberg Hospitals, The Capital \\ Region, 2400 Copenhagen, Denmark \\ ${ }^{3}$ National Institute of Public Health, University of Southern Denmark, 1353 Copenhagen, Denmark \\ ${ }^{4}$ Sydney Medical School, The Boden Institute of Obesity, Nutrition, Exercise E Eating Disorders, University of Sydney, Sydney, \\ NSW 2006, Australia
}

(Submitted 6 March 2015 - Final revision received 9 July 2015 - Accepted 10 July 2015 - First published online 2 September 2015)

\section{Abstract}

The aim of the present study was to examine the associations of sugary drink consumption and its substitution with alternative beverages with body weight gain among young children predisposed to future weight gain. Secondary analysis of the Healthy Start Study, a 1.5-year randomised controlled trial designed to prevent overweight among Danish children aged 2-6 years ( $n$ 366), was carried out. Multivariate linear regression models were used to investigate the associations of beverage consumption with change in body weight ( $\Delta$ weight) or BMI ( $\Delta$ BMI) $z$-score. Substitution models were used to extrapolate the influence of replacing sugary drinks with alternative beverages (water, milk and diet drinks) on $\Delta$ weight or $\Delta$ BMI $z$-score. Sugary drink intake at baseline and substitution of sugary drinks with milk were associated with both $\Delta$ weight and $\Delta$ BMI $z$-score. Every $100 \mathrm{~g} / \mathrm{d}$ increase in sugary drink intake was associated with $0 \cdot 10 \mathrm{~kg}$ and $0 \cdot 06$ unit increases in body weight $(P=0.048)$ and BMI $z$-score $(P=0.04)$, respectively. Substitution of $100 \mathrm{~g} / \mathrm{d}$ sugary drinks with $100 \mathrm{~g} / \mathrm{d}$ milk was inversely associated with $\Delta$ weight $(\beta=-0.16 \mathrm{~kg} ; P=0.045)$ and $\Delta$ BMI $z$-score $(\beta=-0.07$ units; $P=0.04)$. The results of this study suggest that sugary drink consumption was associated with body weight gain among young children with high predisposition for future overweight. In line with the current recommendations, sugary drinks, whether high in added or natural sugar, should be discouraged to help prevent childhood obesity. Milk may be a good alternative to sugary drinks with regard to weight management among young obesity-predisposed children.

Key words: Sugary drinks: Milk: Young children: Predisposition: Body weight: Obesity

Childhood obesity is an alarming public health issue worldwide $^{(1,2)}$. Beyond its immediate health consequences among children and adolescents, childhood obesity is also a significant risk factor for obesity and a range of medical and psychosocial comorbidities in adulthood ${ }^{(3)}$. Energy imbalance attributable to an increase in energy intake and a decrease in energy expenditure is believed to be the major underlying factor $^{(4)}$. In particular, increased consumption of beverages, particularly those high in sugar, is thought to be one of the potential contributors to the rapid increase in energy intake among children and adolescents ${ }^{(5)}$. Energy from beverages contributes more than $20 \%$ of total energy intake to the diets of children and adolescents in many countries worldwide, such as the UK, Australia and Brazil ${ }^{(6-8)}$. It has been postulated that energy consumed in liquid form has a low satiety value, and may not trigger the physiological satiety mechanisms to the same extent as energy consumed in solid form, resulting in inadequate compensatory reduction in energy from other food sources and subsequently higher energy intake ${ }^{(9)}$. Independent of energy intake, accumulating evidence suggests that large amounts of added sugars in sugar-sweetened beverages (SSB) including carbonated soft drinks and fruit-flavoured drinks may increase obesity risk by contributing to a high glycaemic load and exacerbating insulin response ${ }^{(10)}$. Systematic reviews and meta-analyses of prospective cohort studies and randomised controlled trials (RCT) support a positive association between SSB consumption and weight gain ${ }^{(11,12)}$. Fruit juice contains similar amounts of sugar and energy and may have the same potential to promote body weight gain ${ }^{(13,14)}$. Evidence of a positive association between fruit juice intake and obesity has been reported among young children who were either overweight or at risk of overweight at baseline ${ }^{(15,16)}$. It is, therefore,

Abbreviations: $\triangle$ BMI, change in BMI; SSB, sugar-sweetened beverages; $\Delta$ weight, change in body weight.

* Corresponding author: M. Zheng, email mzhe4938@sydney.edu.au 
important to examine the contribution of sugary drinks (whether high in added or natural sugar) in promoting childhood obesity. The present study aimed to examine the association between sugary drink consumption and change in body weight or BMI $z$-score in a group of $2-6$-year-old children predisposed to future overweight, and, second, to investigate whether water, milk or diet drinks may be better alternatives to sugary drinks with regard to body weight management using substitution models.

\section{Methods \\ Study population}

The Healthy Start Study is a $1 \cdot 5$-year RCT designed to prevent overweight and obesity among 2-6-year-old Danish children who were normal weight, but had a high predisposition for future overweight, based on one or more of the following risk factors: mothers who were overweight or obese before pregnancy $\left(\mathrm{BMI} \geq 28 \mathrm{~kg} / \mathrm{m}^{2}\right.$ ), low socio-economic status (maternal education $\leq 10$ years) or babies born with a high birth weight $(\geq 4 \mathrm{~kg})^{(17)}$. The intervention strategies included the following four major areas: healthy changes to dietary habits, increasing physical activity, improvement in sleep duration and quality and reduction of chronic stress in the child and the family. The level of intervention was planned and tailored to each participating family's individual needs, based on the information from dietary records and the parental questionnaire. Stages of change principles and motivational interviewing were used as the framework for the counselling process.

In 2009, data on birth weight and pre-pregnancy BMI of mothers of all the children born between 2004 and 2007 in eleven selected municipalities from the greater Copenhagen area were obtained from the Danish National Birth Register at the National Board of Health. Data on socio-economic status (estimated from maternal education level) were obtained from the administrative birth forms filled out by hospital personnel at the time of birth. Children were excluded before randomisation if they had moved to another municipality after they were born, if they were protected from being contacted by researchers, if they did not have a permanent address, lived in a children's home, had died or moved abroad. Children eligible for participation were allocated to an intervention group or a control group using computer-based randomisation. Children in the intervention group were followedup by the same health consultant throughout the project period. Children in the control group were invited to see a health consultant only at baseline and at follow-up visits. The intervention ended in the summer of 2011. A detailed study protocol has been published ${ }^{(17)}$ and approved by the Danish Data Protection Agency (journal number: 2007-41-0530). Informed consent to use the collected data for research purpose was obtained from all the participants' parents.

The present analyses included children ( $n$ 366) from both intervention and control groups who participated in both baseline and follow-up examinations over 1.5 years (Fig. 1). Children who misreported their energy intake at baseline ( $n 14)$ and at follow-up ( $n$ 16) were excluded. A further forty-eight children were excluded from the longitudinal analysis due to incomplete dietary data at follow-up. As a result, a final number of 352 and 288 children were included in the prospective (baseline beverage intake and change in body weight) and longitudinal (concurrent change in beverage intake and change in body weight) analyses, respectively. Misreporting of energy intake at either baseline or at follow-up was evaluated using the Goldberg cut-off method by calculating the ratio of reported energy intake:estimated energy requirements ${ }^{(18)}$. The individual estimated energy requirements were assessed using energy requirement equations for children and adolescents proposed by the FAO/WHO/UN University expert consultation report ${ }^{(19)}$.

\section{Measurement of dietary and beverage intake}

A 4-d dietary record was completed by parents to capture dietary intake of their child on both weekdays and weekends. Along with the dietary record, a picture booklet was provided to assist parents with estimating portion sizes. Each dietary record was entered into a software program (Dankost 3000) for food and nutrient analysis. Daily intakes were calculated as the average of 4-d records. Beverages were classified as (1) water (tap water, sparkling water and still water), (2) milk (skimmed milk, low-fat milk, whole milk, butter milk and flavoured milk), (3) sugary drinks (sugar-sweetened carbonated and fruit-flavoured drinks and fruit juice) and (4) diet drinks (artificially sweetened beverages). Per capita consumption of each beverage category was calculated as grams per $\mathrm{d}$. Total beverage intake was calculated as the sum of water, milk, sugary drink and diet drink intakes.

\section{Measurement of anthropometrics}

Height was measured barefoot or in stockings to the nearest $0.1 \mathrm{~cm}$ using a stature meter (Soehnle Professional 5002 or Charter ch200P). Body weight was measured in underwear to the nearest $0 \cdot 1 \mathrm{~kg}$ using a mechanical weight or a beam-scaletype weight (Tanita BWB-800 or SV-SECA 710). Age- and sex-specific BMI $z$-scores were calculated using the Lambda$\mathrm{Mu}$-Sigma method ${ }^{(20)}$, and Danish national reference $z$-scores were applied to the study population ${ }^{(21)}$.

\section{Covariates}

Information on the number of siblings living with the child, whether or not the parents are divorced, the child's physical activity level, paternal and maternal education level and household income was obtained from a parental questionnaire completed at baseline. The child's physical activity level compared with peers was categorised as low (reported 'not as active' or 'fairly active') or high (reported as 'very active'). Maternal and paternal education levels were classified as lower than tertiary education (primary school, high school, formal and non-formal education or skilled worker) or as tertiary education or above (tertiary education, bachelor level, graduate level or PhD level).

\section{Statistical analysis}

All the statistical analyses were performed using SPSS version 20.0 (SPSS IBM) with statistical significance set at $P<0 \cdot 05$, two-tailed. Descriptive analyses were carried out at baseline and at follow-up. Multivariable linear regression was used to examine the 
(a)

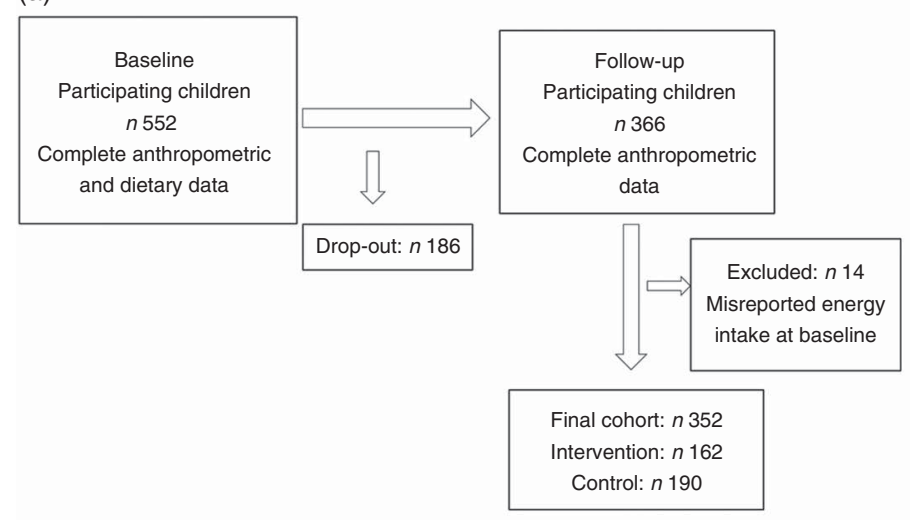

(b)

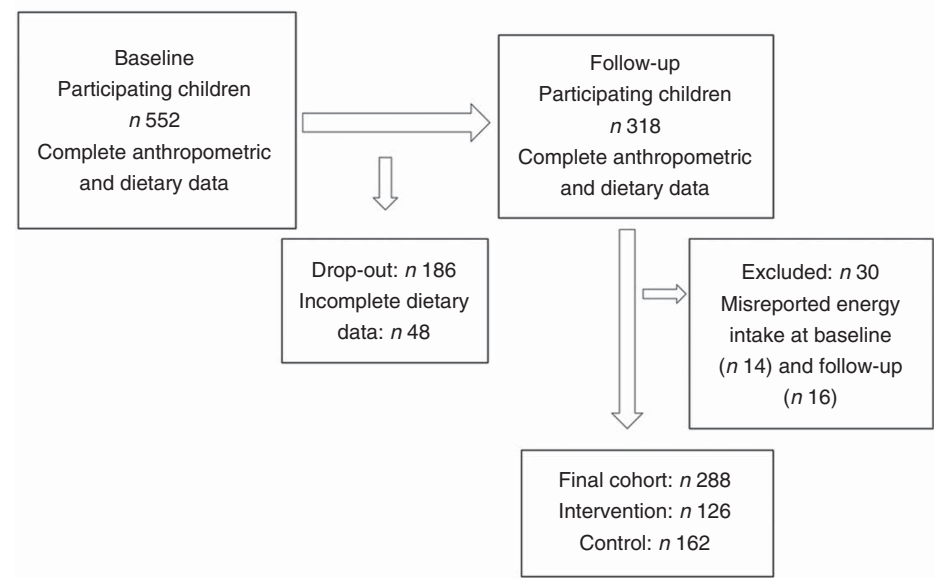

Fig. 1. Flow chart showing participation of the study population: (a) children included in the prospective analysis (association between baseline beverage intake and change in body weight), (b) children included in the longitudinal analysis (association between change in beverage intake and change in body weight).

associations between beverage intake (sugary drinks, water, milk and diet drinks, $100 \mathrm{~g} / \mathrm{d}$, exposure variables) at baseline and change in body weight ( $\Delta$ weight) or BMI $(\Delta \mathrm{BMI}) z$-score (follow-up minus baseline, outcome variables). Additional analyses were carried out to examine the associations between concurrent changes in beverage intake and $\Delta$ weight or $\Delta \mathrm{BMI}$ $z$-score. Separate models were constructed for $\Delta$ weight and $\Delta \mathrm{BMI}$ $z$-score. In order to assess the independent role of individual beverages, all beverage types were included simultaneously in the same model with adjustment for baseline age, BMI $z$-score, sex, intervention allocation, physical activity, whether parents were divorced, number of siblings living with the child, annual income, maternal and paternal educational levels and maternal pre-pregnancy overweight. Potential effect modifications by sex and intervention allocation were tested using stratified analyses. Testing for differences between stratum-specific regression coefficients using the likelihood ratio test was not significant $(P>0.50)$; therefore, all the subjects were analysed together. In models with change in beverage intake as the exposure variable, baseline beverage intake was also included as a covariate. Two energy adjustment methods were used to elucidate the potential mediating effects of energy on the association between beverage intake and obesity. The nutrient residual model included beverage intake residuals along with total energy intake. Beverage intakes (dependent variable) were regressed on total energy intake (independent variable) to create the residuals. This model represents the generic non-energy effects of individual beverage types on changes in body weight ${ }^{(22,23)}$. The energy partition model included the absolute amount of individual beverage intake $(100 \mathrm{~g} / \mathrm{d})$ and energy from non-beverage sources, which allowed the estimation of both specific energy and non-energy effects of individual beverage types ${ }^{(22,23)}$.

The influence of replacing sugary drinks with water, milk or diet drinks at baseline on $\Delta$ weight and $\Delta$ BMI $z$-score were assessed using substitution models ${ }^{(24,25)}$. Substitution models are analogous to energy adjustment models that adjust for total energy intake. However, compared with energy adjustment models, substitution models provide better insights into the health implications of changes in diet or specific replacement of dietary exposures - for example, total energy is comprised of carbohydrate, protein and fat.

Energy adjustment model: outcome $=\beta_{1^{\prime}} \times$ carbohydrate

$$
+\beta_{2^{\prime}} \times \text { total energy intake, }
$$

Substitution model: outcome $=\beta_{1^{\prime \prime}} \times$ carbohydrate

$$
+\beta_{2^{\prime \prime}} \times \text { fat }+\beta_{3^{\prime \prime}} \times \text { total energy intake. }
$$


The energy adjustment model includes carbohydrate as the exposure variable along with total energy intake. The coefficient for carbohydrate $\left(\beta_{1^{\prime}}\right)$ represents the effects of substituting a certain amount of carbohydrate for the same amount of energy from non-carbohydrate sources (fat and protein), while holding constant the intake of total energy. However, this model is unspecific regarding the substitution of carbohydrates. The substitution model includes carbohydrate, fat and total energy intake, whereas the protein as the reference category is excluded from the model. When intakes of total energy and fat are held constant, an increase in carbohydrate implies a corresponding decrease in protein. Thus, the coefficient for carbohydrate $\left(\beta_{1^{\prime \prime}}\right)$ in the substitution model represents the effects of substituting a certain amount of carbohydrate for the same amount of energy from protein on outcome. Likewise, the coefficient of fat $\left(\beta_{2^{\prime \prime}}\right)$ has the same substitution meaning that is, the amount of fat that is substituted for the same amount of energy from protein when carbohydrate and total energy intake are held constant. However, the application of substitution models is not restricted to hold energy intake constant $^{(24,25)}$. When the total amount of one dietary factor is held constant, the substitution effects between the subcomponents of this dietary factor can be manipulated.

In the present analysis, total beverage intake consisted of water, milk, diet drinks and sugary drinks. The substitution model examining the effects of substituting water, milk or diet drinks for sugary drinks can be expressed as follows:

Outcome $=\beta_{1^{\prime \prime \prime}} \times$ total beverage $(100 \mathrm{~g} / \mathrm{d})+\beta_{2^{\prime \prime \prime}}$

$$
\begin{aligned}
& \times \text { water }(100 \mathrm{~g} / \mathrm{d})+\beta_{3^{\prime \prime \prime}} \times \operatorname{milk}(100 \mathrm{~g} / \mathrm{d})+\beta_{4^{\prime \prime \prime}} \\
& \times \operatorname{diet} \operatorname{drinks}(100 \mathrm{~g} / \mathrm{d})+\text { covariates },
\end{aligned}
$$

where $\beta_{1^{\prime \prime \prime}}-\beta_{4^{\prime \prime \prime}}$ are regression coefficients.

The substitution model included absolute intakes of total beverages and individual beverage alternatives $(100 \mathrm{~g} / \mathrm{d})$ for sugary drinks. Sugary drinks as the reference category was excluded from the substitution model. By forcing total beverage intake and intakes of milk and diet drinks to be constant, an increase in water intake by definition statistically results in a corresponding decrease in sugary drink intake. The coefficient of milk can be interpreted as the effect of substituting a certain amount of milk for the same amount of sugary drinks. Similarly, the coefficients of water and diet drinks have the same substitution interpretation. The substitution models were adjusted for all the covariates noted above. Further adjustment for energy intake from beverages was conducted to examine whether beverage energy mediated or explained the substitution effects.

\section{Results}

Characteristics of children who participated in both baseline and follow-up examinations ( $n$ 352) are presented in Table 1 . In all, $55 \%$ of the participants were boys, $46 \%$ came from the intervention group, $59 \%$ had high physical activity level, $6 \%$ of the participants' parents were divorced, $78 \%$ of the mothers and $61 \%$ of fathers had an educational level of tertiary degree or above and $46 \%$ of mothers were overweight before pregnancy. As expected, height, body weight and BMI $z$-scores increased during the study period $(P<0 \cdot 0001)$.

Nutrient and beverage intakes at baseline and follow-up are shown in Table 2. From baseline to follow-up, intakes of total energy, fat, protein and carbohydrates increased significantly $(P<0 \cdot 01)$. Similarly, total beverage intake as well as intake of water and diet drinks increased from baseline to follow-up $(P<0.05)$. However, sugary drinks and milk intakes at baseline were not significantly different from the intakes at follow-up $(P>0.05)$.

\section{Beverage consumption and changes in body weight or BMI z-score}

Results of the regression analyses conducted to assess the prospective associations of water, milk, sugary drinks and diet drinks in relation to $\Delta$ weight and $\Delta$ BMI $z$-score are shown in Table 3. Sugary drink intake at baseline was significantly associated with $\Delta$ weight $(\beta=0.1 \mathrm{~kg} ; P=0.048)$ and $\Delta \mathrm{BMI}$

Table 1. Characteristics of participants who participated in both baseline and follow-up examinations

\begin{tabular}{|c|c|c|c|c|c|}
\hline & \multicolumn{2}{|c|}{ Baseline } & \multicolumn{2}{|c|}{ Follow-up } & \multirow[b]{2}{*}{$P^{\star}$} \\
\hline & Mean & SD & Mean & SD & \\
\hline Age (years) & $4 \cdot 1$ & $1 \cdot 1$ & 5.4 & $1 \cdot 1$ & $<0.001$ \\
\hline Height $(\mathrm{cm})$ & 105 & $9 \cdot 4$ & 11.4 & $8 \cdot 9$ & $<0.001$ \\
\hline Body weight $(\mathrm{kg})$ & $18 \cdot 0$ & 3.3 & $21 \cdot 0$ & $3 \cdot 8$ & $<0.001$ \\
\hline BMI z-score (units) & 0.3 & 0.9 & 0.4 & 0.9 & $<0.001$ \\
\hline Males (\%) & \multicolumn{2}{|c|}{54.8} & & & \\
\hline Intervention group (\%) & \multicolumn{2}{|c|}{$46 \cdot 0$} & & & \\
\hline Physical activity, high (\%) & \multicolumn{2}{|c|}{$59 \cdot 2$} & & & \\
\hline Parents divorced $(\%)$ & \multicolumn{2}{|c|}{$5 \cdot 6$} & & & \\
\hline Number of siblings living with the child & $1 \cdot 1$ & 0.7 & & & \\
\hline Maternal education level, tertiary education or above (\%) & \multicolumn{2}{|c|}{$78 \cdot 0$} & & & \\
\hline Paternal education level, tertiary education or above (\%) & \multicolumn{2}{|c|}{$61 \cdot 0$} & & & \\
\hline Maternal pre-pregnancy overweight (\%) & \multicolumn{2}{|c|}{$46 \cdot 3$} & & & \\
\hline
\end{tabular}
(Mean values and standard deviations; $n$ 352)

* Paired $t$ test. 
Table 2. Mean daily nutrient and beverage intakes at baseline and at follow-up* (Mean values and standard deviations; $n$ 288)

\begin{tabular}{|c|c|c|c|c|c|c|c|}
\hline & \multicolumn{2}{|c|}{ Baseline } & \multicolumn{2}{|c|}{ Follow-up } & \multicolumn{2}{|c|}{$\Delta$} & \multirow[b]{2}{*}{$P+$} \\
\hline & Mean & SD & Mean & SD & Mean & SD & \\
\hline \multicolumn{8}{|l|}{ Nutrient intakes } \\
\hline Total energy (MJ) & 4.97 & 0.95 & 5.57 & 0.11 & 0.5 & $1 \cdot 2$ & $<0.0001$ \\
\hline Fat $(\mathrm{g})$ & $39 \cdot 3$ & 11.4 & $45 \cdot 6$ & 13.5 & $6 \cdot 3$ & 14.0 & $<0.0001$ \\
\hline Protein (g) & $45 \cdot 2$ & $10 \cdot 9$ & $50 \cdot 6$ & 13.4 & 5.4 & $13 \cdot 9$ & $<0.0001$ \\
\hline Carbohydrate (g) & $166 \cdot 6$ & $35 \cdot 6$ & 180.5 & $40 \cdot 8$ & 13.9 & $39 \cdot 7$ & $<0.0001$ \\
\hline \multicolumn{8}{|l|}{ Beverage intake (q) } \\
\hline Sugary drinks & $92 \cdot 0$ & $107 \cdot 0$ & $90 \cdot 1$ & $108 \cdot 7$ & -1.9 & $110 \cdot 5$ & 0.76 \\
\hline Water & 89.6 & 174.5 & 211.6 & 194.4 & 22 & $197 \cdot 3$ & 0.048 \\
\hline Milk & 259.6 & $167 \cdot 5$ & $270 \cdot 4$ & $163 \cdot 1$ & $10 \cdot 9$ & 178.6 & 0.28 \\
\hline Diet drinks & 24.8 & 65.0 & 37.6 & 79.8 & $12 \cdot 9$ & 78.0 & 0.004 \\
\hline Total beverage & 537.4 & 300.9 & 615.9 & 291.4 & 42.5 & 303.6 & 0.01 \\
\hline
\end{tabular}

$\Delta$, Change.

* Sugary drinks: carbonated drinks, fruit-flavoured drinks and fruit juices.

$\dagger$ Paired $t$ test.

Table 3. Associations between intakes of sugary drinks, water, milk and diet drinks with changes $(\Delta)$ in body weight or BMI $z$-score over 1.5-year follow-up*

( $\beta$ Coefficients and their standard errors)

\begin{tabular}{|c|c|c|c|c|c|c|}
\hline & \multicolumn{3}{|c|}{$\Delta$ Weight $(\mathrm{kg})$} & \multicolumn{3}{|c|}{$\Delta \mathrm{BMI} z$-score } \\
\hline & $\beta$ & SE & $P$ & $\beta$ & SE & $P$ \\
\hline \multicolumn{7}{|c|}{ Sugary drinks $(100 \mathrm{~g} / \mathrm{d})$} \\
\hline Model 1† & 0.10 & 0.07 & 0.048 & 0.06 & 0.03 & 0.04 \\
\hline Model $2 \ddagger$ & 0.10 & 0.07 & 0.08 & 0.05 & 0.03 & 0.10 \\
\hline Model $3 \S$ & $0 \cdot 10$ & 0.07 & 0.05 & 0.06 & 0.03 & 0.04 \\
\hline \multicolumn{7}{|c|}{ Water $(100 \mathrm{~g} / \mathrm{d})$} \\
\hline Model $1 \dagger$ & 0.06 & 0.04 & 0.16 & 0.01 & 0.03 & 0.66 \\
\hline Model $2 \ddagger$ & 0.06 & 0.04 & 0.17 & 0.01 & 0.02 & 0.62 \\
\hline \multicolumn{7}{|l|}{ Milk (100 g/d) } \\
\hline Model $1 \dagger$ & -0.05 & 0.04 & 0.17 & -0.01 & 0.02 & 0.63 \\
\hline Model $2 \ddagger$ & -0.07 & 0.05 & 0.13 & -0.02 & 0.02 & 0.41 \\
\hline Model 3§ & -0.07 & 0.04 & 0.20 & -0.01 & 0.02 & 0.66 \\
\hline \multicolumn{7}{|c|}{ Diet drinks $(100 \mathrm{~g} / \mathrm{d})$} \\
\hline Model $1 \dagger$ & -0.11 & 0.15 & 0.46 & -0.10 & 0.07 & 0.12 \\
\hline Model $2 \ddagger$ & -0.09 & 0.15 & 0.53 & -0.09 & 0.07 & 0.15 \\
\hline Model 3§ & $-0 \cdot 10$ & 0.15 & 0.48 & $-0 \cdot 10$ & 0.07 & 0.14 \\
\hline
\end{tabular}

* Sugary drinks: carbonated drinks, fruit-flavoured drinks and fruit juices.

$\dagger$ All beverages were included simultaneously in the same model with adjustment for baseline age, BMI $z$-score, sex, intervention allocation, physical activity, whether parents were divorced, number of siblings living with the child, annual income, maternal education level, paternal education level and maternal pre-pregnancy overweight.

‡ Nutrient residual model included beverage intake residuals with adjustment for total energy intake and confounders in model 1.

$\S$ Energy partition model with further adjustment for energy intake from non-beverage sources on model 1. Water without energy was excluded from the energy partition model.

$z$-score $(\beta=0.06$ units; $P=0.04)$ over 1.5 years. After adjusting for total energy intake at baseline in the residual model, the associations between sugary drink intake and $\Delta$ weight $(\beta=0.10 \mathrm{~kg} ; \quad P=0.08)$ and $\Delta$ BMI $z$-score $(\beta=0.05$ units; $P=0 \cdot 10)$ were no longer significant. In the energy partition model, after adjusting for energy from non-beverage sources, the estimated associations between sugary drink intake and $\Delta$ weight $(\beta=0.10 \mathrm{~kg} ; P=0.05)$ and $\Delta \mathrm{BMI}$ z-score $(\beta=0.06$ units; $P=0.04)$ remained similar. No evidence of an association was found for other beverage types. Regression analyses results
Table 4. Regression analysis results for association between substitution of sugary drinks with water, milk and diet drinks $(100 \mathrm{~g} / \mathrm{d})$ at baseline and changes $(\Delta)$ in body weight or BMl $z$-score over 1.5-year follow-up ( $\beta$ Coefficients and their standard errors)

\begin{tabular}{|c|c|c|c|c|c|c|}
\hline & \multicolumn{3}{|c|}{$\Delta$ Weight (kg) } & \multicolumn{3}{|c|}{$\Delta \mathrm{BMI} z$-score } \\
\hline & $\beta$ & SE & $P$ & $\beta$ & SE & $P$ \\
\hline \multicolumn{7}{|c|}{ Water $(100 \mathrm{~g} / \mathrm{d})$} \\
\hline Model $1^{*}$ & -0.04 & 0.09 & 0.61 & -0.06 & 0.04 & 0.15 \\
\hline Model $2 \dagger$ & 0.02 & 0.08 & 0.69 & 0.08 & 0.06 & 0.19 \\
\hline \multicolumn{7}{|c|}{ Milk $(100 \mathrm{~g} / \mathrm{d})$} \\
\hline Model $1^{*}$ & -0.16 & 0.08 & 0.045 & -0.07 & 0.03 & 0.04 \\
\hline Model $2 \dagger$ & -0.23 & 0.08 & 0.007 & -0.11 & 0.04 & 0.003 \\
\hline \multicolumn{7}{|c|}{ Diet drinks $(100 \mathrm{~g} / \mathrm{d})$} \\
\hline Model $1^{*}$ & -0.21 & 0.17 & 0.22 & -0.17 & 0.07 & 0.03 \\
\hline Model $2 \dagger$ & 0.02 & 0.18 & 0.89 & 0.07 & 0.08 & 0.04 \\
\hline \multicolumn{7}{|c|}{$\begin{array}{l}\text { * Substitution model included water intake }(100 \mathrm{~g} / \mathrm{d}) \text {, milk intake }(100 \mathrm{~g} / \mathrm{d}) \text {, diet drink } \\
\text { intake }(100 \mathrm{~g} / \mathrm{d}) \text { and total beverage intake }(100 \mathrm{~g} / \mathrm{d}) \text {, and excluded sugary drink } \\
\text { intake from the model (reference category). By keeping the intakes of total } \\
\text { beverages, milk and diet drinks constant, a unit increase in water implies a } \\
\text { corresponding decrease in sugary drinks. Model adjusted for baseline age, sex, } \\
\text { BMl } z \text {-score, intervention allocation, physical activity, whether parents were } \\
\text { divorced, number of siblings living with the child, annual income, maternal } \\
\text { education level, paternal education level and maternal pre-pregnancy overweight. } \\
\text { t Adjusted further for energy from beverages on model } 1 \text {. }\end{array}$} \\
\hline
\end{tabular}

for longitudinal associations of changes in water, milk, sugary drink and diet drink intakes with concurrent $\Delta$ weight and $\Delta \mathrm{BMI}$ $z$-scores were not significant (data not shown). Further analyses stratified by intervention allocation were conducted and are presented as online Supplementary Material.

\section{Substitutions of sugary drinks with water, milk or diet drinks at baseline in relation to changes in body weight or BMI z-score}

Replacement of $100 \mathrm{~g} / \mathrm{d}$ sugary drinks with $100 \mathrm{~g} / \mathrm{d}$ milk at baseline was inversely and significantly associated with $\Delta$ weight $(\beta=-0.16 \mathrm{~kg} ; P=0.045)$ and $\Delta$ BMI $z$-score $(\beta=-0.07$ units; $P=0.04$ ) (Table 4). Similarly, replacing $100 \mathrm{~g} / \mathrm{d}$ sugary drinks with $100 \mathrm{~g} / \mathrm{d}$ diet drinks also indicated an inverse association with $\Delta$ BMI $z$-score $(\beta=-0.17$ units; $P=0.03)$. 
No significant associations were found when water was substituted for sugary drinks, although regression coefficients were negative before energy adjustment from beverages.

\section{Discussion}

In this cohort of 2-6-year-old Danish children predisposed to future overweight, sugary drink consumption at baseline was related to subsequent 1.5-year changes in both weight and BMI $z$-scores. Every $100 \mathrm{~g}$ difference in sugary drink intake was associated with a $0.10 \mathrm{~kg}$ and 0.06 unit differences in body weight and BMI $z$-score, respectively. Furthermore, substitution of sugary drinks with milk or diet drinks demonstrated significant beneficial effects on changes in both weight and BMI $z$-score. No significant associations were found between concurrent changes in beverage intakes and change in body weight or BMI $z$-score.

Consistent with our finding, Welsh et $a l^{(15)}$ found that children aged 2-3 years who were at risk for overweight at baseline and consumed one or more servings of sugary drinks including both SSB and fruit juice were significantly more likely to become overweight at 1-year follow-up than those who were normal weight at baseline (odds range: 1.7-1.9). Three more studies among young children reported positive associations between SSB consumption and obesity outcomes ${ }^{(23-25)}$. In a large cohort of US children, children drinking SSB daily at the age of 2 years exhibited greater increase in BMI $z$-score at the age of 5 years than infrequent or non-consumers ${ }^{(26)}$. Likewise, each additional ounce of SSB intake at 3-5 years of age significantly increased the odds of becoming overweight by $4 \%$ at the 2 -year follow-up among African-American children ${ }^{(27)}$. In a large US sample, children who consumed SSB during infancy also demonstrated higher odds of obesity at age 6 years when compared with non-consumers ${ }^{(28)}$. In contrast, the study by Newby et $a{ }^{(29)} \mathrm{did}$ not find a significant association between SSB consumption and changes in body weight or BMI in a cohort of 2-5-year-old children over 6-12 months. Failure to observe an association was likely due to the low intake of SSB and shorter follow-up ${ }^{(29)}$.

Several hypotheses have been proposed to link sugary drink consumption and body weight outcomes ${ }^{(30)}$. Our analyses support the hypothesis that total energy intake is a potential mediator in the pathway between sugary drink consumption and body weight outcomes, as adjusting for total energy intake rendered the estimated association non-significant. However, adjustment for energy from non-beverage sources did not substantially alter the estimated associations, indicating that the association is independent of energy intake from non-beverage sources. Therefore, the association is likely a result of the additional energy provided by sugary drinks and/or non-energy effects such as the glycaemic or metabolic effects of sugars such as glucose and fructose. Large amounts of the rapidly absorbable carbohydrates (i.e. glucose) in sugary drinks can contribute to a high dietary glycaemic load. A high glycaemic load diet exacerbates glucose and insulin responses, which have been associated with increased appetite, food intake and body weight gain $^{(30)}$. Hepatic metabolism of fructose favours body weight gain and fat gain through de novo lipogenesis and a decrease in leptin secretion ${ }^{(31)}$.
Over the study period, the consumption of sugary drinks and milk remained similar, whereas the consumption of water and diet drinks increased. This may be a consequence of the intervention for obesity prevention. Despite this, we failed to find significant longitudinal associations between concurrent changes in beverage intake and change in body weight or BMI $z$-score. Limited changes in the diet and changes in body weight beyond the normal growth in the cohort over 1.5 years could be an explanation for this null finding. Longitudinal associations are more likely to be found in studies with longer study duration with more pronounced changes in diet and anthropometrics. This was confirmed in a longitudinal cohort of Danish children, where change in SSB consumption from childhood to adolescence was a significant predictor of subsequent change in body fatness from adolescence to early adulthood ${ }^{(32)}$. Furthermore, a secondary analysis of RCT among adults found a significant positive association between change in sugary drink consumption and weight loss over 18 months. A significant decrease in sugary drink consumption from baseline to followup was observed over the study period ${ }^{(33)}$.

The optimal beverage to replace sugary drinks needs further investigation $^{(34)}$. A secondary analysis of the Stanford A to Z weight loss intervention in adults demonstrated that replacing sugary drinks with water was positively associated with weight loss over 1 year ${ }^{(25)}$. In our cohort of young children, both milk and diet drinks were inversely associated with weight gain and BMI $z$-score when substituted for SSB. We previously found that substitution of SSB with water or milk was inversely associated with subsequent 6-year change in body fatness in a cohort of 9-year-old Danish children ${ }^{(35)}$. Similarly, SSB when replaced by water or diet drinks was also associated with a significant decrease in BMI $z$-score among 8-year-old Australian children $^{(36)}$. Several RCT have also demonstrated that replacing sugary drinks with low-energy beverages (water and/or diet drinks) or milk exhibited beneficial effects on body composition ${ }^{(37-39)}$. Current beverage consumption trends reveal displacement of milk with sugary drinks ${ }^{(40-42)}$. Milk should be promoted in lieu of sugary drinks not only as a rich source of essential nutrients for children's growth and overall health but also for its potential positive effects on body weight regulation. The favourable effects of milk when substituted for sugary drinks on body weight may be attributable to several milk constituents such as $\mathrm{Ca}$, conjugated linoleic acids and milk proteins ${ }^{(43)}$. It has to be noted that reduced fat and skim milk contributed to the majority (approximately 90\%) of milk intake by this population, and the proportion of flavoured milk in total milk intake was small (2\%). Diet drinks as an alternative option to sugary drinks needs further consideration, especially for children. Although these drinks may be offered to children by parents concerned about their child's weight ${ }^{(44)}$, they are not recommended for young children due to the presence of artificial sweeteners, caffeine and their high acidity level ${ }^{(45,46)}$. Dental erosion is a particular concern in young children and adolescents. Water is the first beverage choice recommended by national guidelines ${ }^{(47,48)}$. However, we found no association for water when substituted for sugary drinks. This is likely a consequence of under-reporting of water intake in dietary records ${ }^{(49)}$, as water consumption is a highly variable event and 
its consumption during the day or unaccompanied with foods is often neglected in dietary records ${ }^{(49)}$. Given that the mean water intake at both baseline and at follow-up was less than a cup, under-reporting of water intake in our study was likely.

Our study has several strengths. First, we contemplated the obesogenic effects of sugary drinks as well as other commonly consumed beverage types. Second, the anthropometric measurements were taken by study investigators rather than being self-reported, which mitigates reporting bias. Third, we were able to collect the absolute amounts of beverage consumed over $4 \mathrm{~d}$ rather than the frequency of consumption. Fourth, our multivariate linear regression model adjusted for a range of confounding factors such as age, baseline BMI $z$-score, parental education level, physical activity and several other family-related factors. Fifth, utilisation of two energy adjustment models allowed for better understanding of both energy and non-energy effects of sugary drink consumption on changes in body weight. Finally, utilisation of substitution modelling allows us to assess the effect of substituting one beverage type for another.

Limitations of this study include the possibility of parental misreporting of dietary intake. Indeed, a national report on sugar habits of 4-12-year-old Danish children indicated that children actually consumed more sugar than that their parents were aware of ${ }^{(50)}$. Although the extreme misreporters were excluded, potential random and systematic reporting bias cannot be ruled out. Our study included a large proportion of pre-pregnancy overweight mothers, and systematic under-reporting may be more common in this population for foods and beverages considered 'unhealthy'(51). This may bias the results and move the association towards null. However, we were still able to observe a significant association, suggesting that the true association might be even stronger. The present study included only body weight and BMI, which are crude measures of adiposity in children. Other anthropometric measures such as body fat, lean mass and skinfolds are warranted in future studies. The generalisability of our study to the general population of young children is limited as our study consisted of 2-6-year-old children predisposed to overweight. Finally, the nature of the study cannot infer causal relationships, and only associations can be derived. Other unmeasured or residual confounding factors may be present and have an impact on the results.

\section{Conclusion}

Sugary drink consumption was associated with body weight gain among young normal weight children with high predisposition for future overweight. In line with current recommendations, sugary drinks, whether high in added or natural sugar, should be discouraged to help prevent childhood obesity. A $0.1 \mathrm{~kg}$ increase in weight gain for every $100 \mathrm{~g} / \mathrm{d}$ of sugary drinks over 1.5 years can translate to an additional $0.15 \mathrm{~kg}$ weight gain for 4-6-year-old Danish children who consumed an average of $150 \mathrm{~g} / \mathrm{d}$ sugary drinks ${ }^{(40)}$. As sugary drink consumption is only one of many factors that can contribute to weight gain and is easily modifiable, it is important to inform the general public to limit sugary drink consumption in an early age. Milk was shown to be a good alternative to sugary drinks with regard to body weight management.
Considering the displacement of milk by sugary drinks among children in recent years, it is also prudent to encourage children to consume more milk. The results of the present study suggest that body weight status of children with high predisposition to weight gain, whether biological or social in origin, is modifiable. Advising parents of obesity-predisposed young children to replace sugary drinks with milk may be helpful for obesity prevention in such groups.

\section{Acknowledgements}

This research received no specific grant from any funding agency, commercial or not-for-profit sectors.

B. L. H. and J. F. R. collected the data. M. Z. conceptualised and designed the research, performed statistical analysis, interpreted the data and wrote the manuscript. A. R. and B. L. H. assisted with the conception of the research, interpretation of data and manuscript writing. M. A.-F., N. J. O. and J. F. R. assisted with interpretation of the data and manuscript writing. All the authors critically reviewed and approved the final version of the manuscript.

There are no conflicts of interest to declare.

\section{Supplementary material}

For supplementary material/s referred to in this article, please visit http://dx.doi.org/10.1017/S0007114515002974

\section{References}

1. Lobstein T, Baur L \& Uauy R (2004) Obesity in children and young people: a crisis in public health. Obes Rev 5, Suppl. 1, 4-104.

2. Adair LS (2008) Child and adolescent obesity: epidemiology and developmental perspectives. Physiol Behav 94, 8-16.

3. Pulgaron ER (2013) Childhood obesity: a review of increased risk for physical and psychological comorbidities. Clin Ther 35, A18-A32.

4. Anderson PM \& Butcher KE (2006) Childhood obesity: trends and potential causes. Future Child 16, 19-45.

5. Craigie AM, Lake AA, Kelly SA, et al. (2011) Tracking of obesity-related behaviours from childhood to adulthood: a systematic review. Maturitas 70, 266-284.

6. Ng SW, Ni Mhurchu C, Jebb SA, et al. (2012) Patterns and trends of beverage consumption among children and adults in Great Britain, 1986-2009. Br J Nutr 108, 536-551.

7. Australian Bureau of Statistics (2014) Australian Health Survey: Nutrition First Results - Foods and Nutrients, 201112. Canberra: Australian Bureau of Statistics.

8. Feferbaum R, de Abreu L \& Leone C (2012) Fluid intake patterns: an epidemiological study among children and adolescents in Brazil. BMC Public Health 12, 1005.

9. Drewnowski A \& Bellisle F (2007) Liquid calories, sugar, and body weight. Am J Clin Nutr 85, 651-661.

10. Bray GA \& Popkin BM (2014) Dietary sugar and body weight: have we reached a crisis in the epidemic of obesity and diabetes?: health be damned! Pour on the sugar. Diabetes Care 37, 950-956.

11. Mattes R, Shikany J, Kaiser K, et al. (2011) Nutritively sweetened beverage consumption and body weight: a systematic review and meta-analysis of randomized experiments. Obes Rev 12, 346-365. 
12. Malik VS, Pan A, Willett WC, et al. (2013) Sugar-sweetened beverages and weight gain in children and adults: a systematic review and meta-analysis. Am J Clin Nutr 98, 1084-1102.

13. Taylor R, Scragg R \& Quigley R (2005) Do sugary drinks contribute to obesity in children? A report prepared by the Scientific Committee of the Agencies for Nutrition Action. Wellington: Agencies for Nutrition Action.

14. Committee on Nutrition (2001) American Academy of Pediatrics: the use and misuse of fruit juice in pediatrics. Pediatrics 107, 1210-1213

15. Welsh JA, Cogswell ME, Rogers S, et al. (2005) Overweight among low-income preschool children associated with the consumption of sweet drinks: Missouri, 1999-2002. Pediatrics, 115, e223-e229.

16. Faith MS, Dennison BA, Edmunds LS, et al. (2006) Fruit juice intake predicts increased adiposity gain in children from lowincome families: weight status-by-environment interaction. Pediatrics 118, 2066-2075.

17. Olsen NJ, Buch-Andersen T, Handel MN, et al. (2012) The Healthy Start project: a randomized, controlled intervention to prevent overweight among normal weight, preschool children at high risk of future overweight. BMC Public Health 12, 590.

18. Black AE (2000) Critical evaluation of energy intake using the Goldberg cut-off for energy intake: basal metabolic rate. A practical guide to its calculation, use and limitations. Int J Obes Relat Metab Disord 24, 1119-1130.

19. Torun B (2005) Energy requirements of children and adolescents. Public Health Nutr 8, 968-993.

20. Cole TJ \& Green PJ (1992) Smoothing reference centile curves: the LMS method and penalized likelihood. Stat Med 11, 1305-1319.

21. Nysom K, Mølgaard C, Hutchings B, et al. (2001) Body mass index of 0 to 45-y-old Danes: reference values and comparison with published European reference values. Int I Obes Relat Metab Disord 25, 177-184.

22. Hu F, Stampfer M, Rimm E, et al. (1999) Dietary fat and coronary heart disease: a comparison of approaches for adjusting for total energy intake and modeling repeated dietary measurements. Am J Epidemiol 149, 531-540.

23. Willett W, Howe G \& Kushi L (1997) Adjustment for total energy intake in epidemiologic studies. Am J Clin Nutr 65 1220S-1228s

24. Willett W (2013) Nutritional Epidemiology, 3rd ed. New York: Oxford University Press.

25. Stookey JD, Constant F, Popkin BM, et al. (2008) Drinking water is associated with weight loss in overweight dieting women independent of diet and activity. Obesity 16, 2481-2488.

26. DeBoer MD, Scharf RJ \& Demmer RT (2013) Sugar-sweetened beverages and weight gain in 2- to 5-year-old children. Pediatrics 132, 413-420.

27. Lim S, Zoellner J, Lee J, et al. (2009) Obesity and sugarsweetened beverages in African-American preschool children: a longitudinal study. Obesity 17, 1262-1268.

28. Pan L, Li R, Park S, et al. (2014) A longitudinal analysis of sugar-sweetened beverage intake in infancy and obesity at 6 years. Pediatrics 134, Suppl. 1, S29-S35.

29. Newby P, Peterson K, Berkey C, et al. (2004) Beverage consumption is not associated with changes in weight and body mass index among low-income preschool children in North Dakota. J Am Diet Assoc 104, 1086-1094.

30. Banchman C, Baranowski T \& Nicklas T (2006) Is there an association between sweetened beverages and adiposity? Nutr Rev 64, 153-174

31. Bray GA \& Popkin BM (2013) Calorie-sweetened beverages and fructose: what have we learned 10 years later. Pediatr Obes 8, 242-248.

32. Zheng M, Rangan A, Olsen NJ, et al. (2014) Sugar-sweetened beverages consumption in relation to changes in body fatness over 6 and 12 years among 9-year-old children: the European Youth Heart Study. Eur J Clin Nutr 68, 77-83.

33. Chen L, Appel L, Loria C, et al. (2009) Reduction in consumption of sugar-sweetened beverages is associated with weight loss: the PREMIER trial. Am J Clin Nutr 89, 1299-1306.

34. Zheng M, Allman-Farinelli M, Heitmann BL, et al. (2015) Substitution of sugar-sweetened beverages with other beverage alternatives: a review of long-term health outcomes. I Acad Nutr Diet 115, 767-779.

35. Zheng M, Rangan A, Olsen NJ, et al. (2014) Substituting sugarsweetened beverages with water or milk is inversely associated with body fatness development from childhood to adolescence. Nutrition 31, 38-44.

36. Zheng M, Allman-Farinelli M, Heitmann BL, et al. (2014) Liquid versus solid energy intake in relation to body composition among Australian children. J Hum Nutr Diet 8, Suppl. 2, 70-79.

37. Albala C, Ebbeling C, Cifuentes M, et al. (2008) Effects of replacing the habitual consumption of sugar-sweetened beverages with milk in Chilean children. Am J Clin Nutr $\mathbf{8 8}$, 605-611.

38. Ebbeling C, Feldman H, Chomitz V, et al. (2012) A randomized trial of sugar-sweetened beverages and adolescent body weight. $N$ Engl J Med 367, 1407-1416.

39. Tate DF, Turner-McGrievy G, Lyons E, et al. (2012) Replacing caloric beverages with water or diet beverages for weight loss in adults: main results of the Choose Healthy Options Consciously Everyday (CHOICE) randomized clinical trial. Am J Clin Nutr 95, 555-563.

40. Fagt S, Biltoft-Jensen A, Matthiessen J, et al. (2008) Dietary habits in Denmark 1995-2006. Copenhagen: National Food Institute, Technical University of Denmark.

41. Duffey K \& Popkin B (2007) Shifts in patterns and consumption of beverages between 1965 and 2002. Obesity 15, 2739-2747.

42. Clifton P, Chan L, Moss C, et al. (2011) Beverage intake and obesity in Australian children. Nutr Metab 8, 87-98.

43. Dougkas A, Reynolds C, Givens I, et al. (2011) Associations between dairy consumption and body weight: a review of the evidence and underlying mechanisms. Nutr Res Rev 15, 1-24.

44. Seburg EM, Kunin-Batson A, Senso MM, et al. (2014) Concern about child weight among parents of children at-risk for obesity. Health Behav Policy Rev 1, 197-208.

45. Sylvetsky A, Rother KI \& Brown R (2011) Artificial sweetener use among children: epidemiology, recommendations, metabolic outcomes, and future directions. Pediatr Clin North Am 58, $1467-1480$.

46. Dietary Guidelines Advisory Committee (2010) Report of the Dietary Guidelines Advisory Committee on the Dietary Guidelines for Americans, 2010, to the Secretary of Agriculture and the Secretary of Health and Human Services. Washington, DC: US Department of Agriculture, Agricultural Research Service.

47. Nordic Council of Ministers (2012) Nordic Nutrition Recommendations 2012: Integrating Nutrition and Physical Activity, 5th ed. Copenhagen: Nordic Council of Ministers.

48. US Department of Agriculture, US Department of Health and Human Services (2010) Dietary Guidelines for Americans, 2010, 7th ed. Washington, DC: US Government Printing Office.

49. Rush EC (2013) Water: neglected, unappreciated and under researched. Eur J Clin Nutr 67, 492-495.

50. Iversen J, Matthiessen J, Fagt S, et al. (2011) Children's Sugar Habits - A Study of Sugar Culture Among Families with Children 4-12-Year-Old Children. Copenhagen: National Food Institute, Department of Health.

51. Lioret S, Touvier M, Balin M, et al. (2011) Characteristics of energy under-reporting in children and adolescents. Br J Nutr 105, 1671-1680. 